\title{
Establishment of a comprehensive indicator to nondestructively analyze watermelon quality at different ripening stages
}

\author{
Shuye Qi**, Shuhui Song ${ }^{\dagger}$, Shengnan Jiang*, Yingrui Chen*, \\ $\mathrm{Wu} \mathrm{Li}{ }^{\dagger}$ and Donghai Han*\& \\ ${ }^{*}$ College of Food Science and Nutritional Engineering \\ China Agricultural University \\ 17 Qinghua East Road, Haidian District \\ Beijing, P. R. China \\ ${ }^{\dagger}$ National Engineering Research Center for Vegetables \\ 50 Zhanghua Street, Haidian District \\ Beijing, P. R. China \\ ¥qsy_ok@126.com \\ \$handh@cau.edu.cn
}

Received 10 July 2013

Accepted 3 August 2013

Published 4 September 2013

\begin{abstract}
Two nondestructive methods based on visible and near-infrared (VIS-NIR) spectroscopy and $\mathrm{X}$-ray image have been used for the evaluation of watermelon quality. The prediction performance based on partial least squares (PLS) by diffuse transmittance measurement (500-1010 nm) was evaluated for chemical quality attributes SSC $(R c=0.903 ; \mathrm{RMSEC}=0.572 \%$ Brix; $R p=0.862 ; \mathrm{RMSEP}=0.717 \%$ Brix; RPD $=1.83)$, lycopene $(R c=0.845 ; \mathrm{RMSEC}=0.266 \mathrm{mg} /$ $100 \mathrm{gFW} ; R p=0.751 ; \mathrm{RMSEP}=0.439 \mathrm{mg} / 100 \mathrm{gFW} ; \mathrm{RPD}=1.13)$ and moisture $(R c=0.917$; $\mathrm{RMSEC}=0.280 \% ; R p=0.937 ; \mathrm{RMSEP}=0.276 \% ; \mathrm{RPD}=2.79)$. The X-ray calibration linear equations developed by extracting the appropriate gray threshold were sufficiently precise for volume $\left(R^{2}=0.986\right)$ and weight $\left(R^{2}=0.993\right)$. In order to optimize prediction model of watermelon quality in growth period, multivariate multi-block technique factor analysis enabled integration of these traits: chemical information is related to physical information. Applying principle component analysis to extract common factors and varimax with Kaiser normalization to improve explanatory, the comprehensive indicator based on variances was established satisfactorily with $R c=0.94, \mathrm{RMSEC}=0.244, R p=0.93, \mathrm{RMSEP}=0.344$ and $\mathrm{RPD}=2.00 . \mathrm{A}$ comparison of these models indicates that the comprehensive indicator determined only by portable VIS-NIR spectrometer appears as a suitable method for appraising watermelon quality nondestructively on the plant at different ripen stages. This method contributes to infer the
\end{abstract}

This is an Open Access article published by World Scientific Publishing Company. It is distributed under the terms of the Creative Commons Attribution 3.0 (CC-BY) License. Further distribution of this work is permitted, provided the original work is properly cited. 
S. Qi et al.

picking date of watermelon with higher accuracy and bigger economic benefits than that by experience.

Keywords: Visible and near-infrared spectroscopy; X-ray imaging; maturity process; factor analysis; comprehensive indicator.

\section{Introduction}

As the fruit matures, its soluble solid content, acidity, dry matter, color, size, firmness and some other properties will change gradually, and each trait practically has been evaluated for harvest quality or eating quality of fruit. ${ }^{1-4}$ The majority of works to assess watermelon quality were destructive and depended on single component. These schemes did not take into account the mutual influence between indicators and considerable detecting time during watermelon maturity. Hence, there is a demand for rapid and new analytical techniques for appraising quality of watermelon.

Near-infrared (NIR) spectroscopy offers a number of significant advantages over traditional methods. It is a noninvasive method with high precision requiring little or no sample preparation, and lower costs. Several determinations can be made simultaneously to reduce time and labor consumption in this multi-analytical technique.

A comparison of acoustic technology, dynamic technology, electrical and magnetic technology, and NIR spectroscopy indicates that online measurement by visible and near-infrared (VIS-NIR) spectroscopy will play an important role in the further study. ${ }^{5}$ In order to realize quality grading rapidly for watermelon nondestructively, the variables were selected by Monte-Carlo uninformative variable eliminate (MC-UVE) in building the partial least squares (PLS) regression model of SSC. ${ }^{6}$ Despite the widespread application, pre-processed spectral data is often not well understood. After investigating the effect of Savitzky-Golay (SG) derivatives (i.e., zero order, first order and second order derivatives) with different filter length, a parsimonious principal component regression (PCR) achieved the best accuracy. ${ }^{7}$ Diffuse reflectance measurements (8002500), physical, physiological and biochemical measurements were performed individually on 877 apricot fruits from eight contrasted cultivars harvested at different ripening stages. Good prediction performance was obtained for $\mathrm{SSC}(R c=0.92$; $\mathrm{RMSEC}=0.99 ; \quad R p=0.92 ; \mathrm{RMSEP}=0.98)$ and titratable $\quad$ acidity $\quad(R c=0.88 ; \quad \mathrm{RMSEC}=3.83$; $R p=0.88 ;$ RMSEP $=3.62)$, while the other quality traits such as firmness $(R c=0.80 ;$ RMSEC $=$ 54.61; $R p=0.74 ;$ RMSEP $=60.27)$, ethylene $(R c=$ $0.86 ; \mathrm{RMSEC}=1.05 ; R p=0.74 ; \mathrm{RMSEP}=60.27$ ), individual sugars and organic acids were with high error of calibration and prediction. ${ }^{8}$ The robustness of total soluble solids, total acidity and firmness models is high in terms of seasonality and range. ${ }^{9}$ The red color imparted to watermelon is due to lycopene who establishing beneficial effects (free-radical scavenger) on human health. Linear correlation $(R=0.917)$ was used to detect the concentration of trans-lycopene in postharvest watermelon. ${ }^{10}$ In our preliminary studies, SSC and moisture were the better indicator to appraise watermelon quality during maturity by VIS-NIR. ${ }^{11}$ And the better location to acquire spectra was on the melon top (calyx) than that to obtain average spectra around the equator. ${ }^{12}$

$\mathrm{X}$-ray is also a nondestructive method which has strong penetration capability. Its digital can be displayed and stored easily, and can be rapidly transferred between applications. Review different methods for nondestructive size determination of horticultural products, focusing on the capable of 3D multispectral scanning to measure fruit volume and weight while neglecting high cost. ${ }^{13}$ The apple volume was calculated by using the ratio between the minus exponential of $\mathrm{X}$-ray gray value and the thickness of apple slice, and the regression correlation coefficients for calibration was $0.9932 .{ }^{14}$

The objective of this study was to establish a more effective and comprehensive indicator to improve prediction accuracy of watermelon quality at different ripening stages. To verify the interrelationship of SSC, lycopene, moisture, volume and weight, each quality trait was measured individually by VIS-NIR or X-ray as two nondestructive methods who were used to compare with the comprehensive model based on factor analysis. And the last comprehensive indicator can be determined only by VIS-NIR portable spectrometer, to evaluate 
internal quality of watermelon and distinguish right picking date on the plant, other than experience.

\section{Methods and Materials}

\subsection{Watermelon samples}

Watermelon, called "Jingxiu" in Beijing, was chosen to determine its fruit traits such as SSC, lycopene, moisture, volume and weight in 2012. To obtain a wide range of fruit composition during the maturation period, about 10 samples for each picking date was collected every two days from $21 \mathrm{~d}$ to $37 \mathrm{~d}$ after pollination, and a total of 94 samples were measured for development of regression models. Noninvasive techniques were performed at the picking day and the reference values were measured destructively within two days at room temperature.

\subsection{VIS-NIR measurement}

The NIR portable spectrometer (Kubota KBA100R, Japan) was used to acquire spectra in diffuse transmittance measurement (500-1010 nm; $2 \mathrm{~nm}$ spectral resolution) with $1 \mathrm{~m}$ fiber optics. To prevent the effect of stray light, the top of watermelon (calyx) was placed in a light-sealed fitting $(d=4 \mathrm{~cm})$ when collecting. Due to ring-shaped light source $(d=3.8 \mathrm{~cm})$ and fixed sampling position (calyx), only one spectrum was collected for each watermelon. The integration times of reference and sample were $50 \mathrm{~ms}$ and $100 \mathrm{~ms}$, respectively.

The main pretreatments to spectra were SG smoothing, first derivative (1D) and second derivative $(2 \mathrm{D})$. SG smoothing was mainly used to eliminate noise, and derivative was performed well to remove the baseline translation and highlight the effective absorption peaks. And all the samples was divided into calibration and validation by almost 3:1 randomly when modeling.

\subsection{X-ray image measurement}

$\mathrm{X}$-ray images were recorded with image capture card (Coreco PC-DIG, Finland) on an X-ray inspection equipment developed by ourselves (showed in Fig. 1).

The voltage and current of X-ray source were adjusted into $80 \mathrm{kV}$ and $1 \mathrm{~mA}$. In order to obtain undistorted image, the integration time of detector

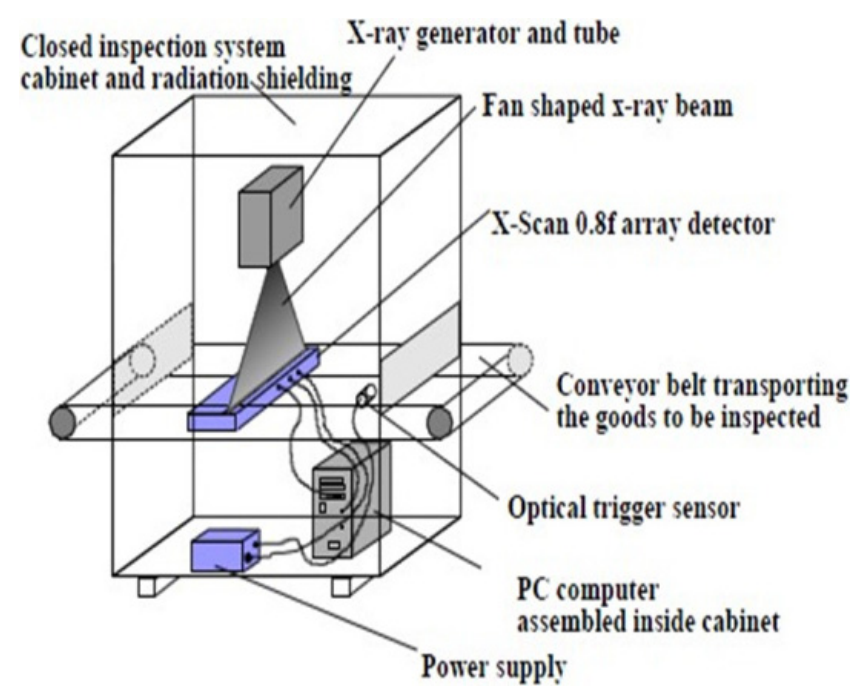

Fig. 1. Diagram of X-ray inspection equipment.

was $2 \mathrm{~ms}$ to match the speed $17.8 \mathrm{~cm} / \mathrm{s}$ of conveyer belt on line. Image data was automatically saved as images (0-255 grayscale) and documents (0-16383 grayscale).

\subsection{Detection of quality traits by reference measurements}

The location to obtain materials for measuring chemical indicators was under calyx rind about $2 \mathrm{~cm} .{ }^{15}$ SSC was detected by Electronic Brix instrument (ATAGO PAL-1, Japan) required to scoop out a piece of flesh about $1 \mathrm{~cm}^{3}$. Lycopene was measured by UV-VIS spectrophotometer (Hitachi U-3410, Japan). The fruits were cut and frozen into powder, then lycopene was extracted by methanol and hexane through the proper cycle of ultrasonic and centrifugation. Moisture was determined by GB8858-88 (Chinese standard). The watermelon block of rind about $50 \mathrm{~g}$ was dehydrated into constant weight at $70-80^{\circ} \mathrm{C}$ and calculated the weight delta. Volume was quantified using displacement method. And weight was measured with high-precision electronic balance (JJ500, USA). The statistical results of five indexes about 94 samples are showed in Table 1.

\subsection{Mathematical analysis of data}

The PLS regression was applied to the normalized spectra after several pretreatment techniques were individually tested to different chemical indicators (SSC, lycopene and moisture). And linear regression 
Table 1. Statistics of chemical and physical parameters of 94 watermelon samples.

\begin{tabular}{lccc}
\hline \multicolumn{1}{c}{ Index } & Range & Mean & SD \\
\hline SSC (\%Brix) & $5.10-11.20$ & 8.45 & 1.36 \\
Lycopene $(\mathrm{mg} / 100 \mathrm{gFW})$ & $0.41-2.60$ & 1.26 & 0.53 \\
Moisture $(\%)$ & $92.62-96.12$ & 94.36 & 0.72 \\
Volume $\left(\mathrm{cm}^{3}\right)$ & $678-1884$ & 1190 & 263 \\
Weight $(\mathrm{kg})$ & $0.621-1.697$ & 1.161 & 0.234 \\
\hline
\end{tabular}

was performed to fit physical indicator (volume and weight) with required gray values. To compare concentration change rule of each index in the growth process, all values were normalized into the same dimension.

In order to optimize prediction model of watermelon quality in growth period, multivariate multiblock technique factor analysis enabled integration of these traits to take into account the interplay of different properties. Principle component analysis was used to extract common factors and varimax with Kaiser normalization was applied to improve factors' explanatory. Finally, the first two PCs of PCA performed on the index values were pooled into one matrix, and this new table was calculated by assigning weight to define the comprehensive indicator. This approach helps to improve the discrimination of different stages of maturity.

The quantitative models were performed in Thermo Scientific TQ Analyst software, and FA was carried out in IBM SPSS Statistics 19. Matlab 2008b and the Unscrambler X 10.0.1 were also used to do statistical treatment in this paper.

\section{Result and Discussion}

Table 2 showed descriptive statistics of quality traits SSC, lycopene, moisture, volume and weight of watermelon samples.

The levels of physic-chemical properties in fruit depended on several factors, including cultivar and season, but mostly on the stage of maturity. ${ }^{16}$ Figure 2 directly showed the slight gradient differences of texture and color. In general, $28 \mathrm{~d}$ after pollination was the time to pick for Jingxiu, which mainly depend on its cultivar. But it always changes due to the particular weather. When watermelon enters maturity stage, it requires reduced water intake, or else it will cause its cracking or illness. In addition, sun-filled environment is better to cultivate watermelons. In June 2012, Beijing had more rainy and cloudy days than previous years, which was responsible for Jingxiu's growth retardation. This cultivar of watermelon was lengthened growth period to 31-33 d after pollination to pick when watermelon do not have ripen completely. With regard to this, Jingxiu has a high respiration to release a certain concentration of carbon dioxide and ethylene in storage that promote watermelon ripening and obtain a higher taste maintained a relatively short time. And $35 \mathrm{~d}$ after pollination can be considered to attain maturity fully.

In order to reveal the changes of the detected contents during ripening period, normalized values of five indicators in Table 2 were presented in the same graph (see Fig. 3), along with the mean values and $95 \%$ confidence intervals.

From $23 \mathrm{~d}$ to $25 \mathrm{~d}$ after pollination, the concentrations of SSC continued to increase reached its maximum $9.85 \%$ brix, except the values of $29 \mathrm{~d}$ and $31 \mathrm{~d}$ decrease slightly according to the heavy rains. As the top of watermelon was the most sensitive location to growth process, the SSC became littler after $35 \mathrm{~d}$ because of the greater respiration. With regard to the higher SSC in $21 \mathrm{~d}$ than $23 \mathrm{~d}$, it may be due to more consumption of nutrient substances for cell division in late days of fruit expanding. Meanwhile the change of volume, directly related to the period of fruit expanding, approved this reason. At the full ripening stage, as expected, the lycopene content sustained growth considerably on account of the abundant amount of light and suitable temperature mainly. ${ }^{17}$ However, the average moisture of rind fell down distinctly into $94 \%$ around $27 \mathrm{~d}$, and remained steady during $27 \mathrm{~d}$ to $37 \mathrm{~d}$. It was relevant to the accumulation of dry matter to a certain extent. During the growth period, weight of watermelon remained steady between $1.154 \mathrm{~kg}$ and $1.297 \mathrm{~kg}$ from $25 \mathrm{~d}$ to $35 \mathrm{~d}$. And the situation of volume was very similar with weight. Consequently, it can be concluded that the density kept stable from the end of fruit enlargement to maturity, and there were no voids inside samples. In addition, the stable location to acquire single spectrum of each sample was on the top of melon using ring-shaped light source $(d=3.8 \mathrm{~cm})$. Consequently the VISNIR spectra were not sensitive to the changes of volume and weight. However, it was easy to detect these two indexes by rapid X-ray image technology, which took a photo of the whole area. In this respect, the comprehensive model just by NIR 


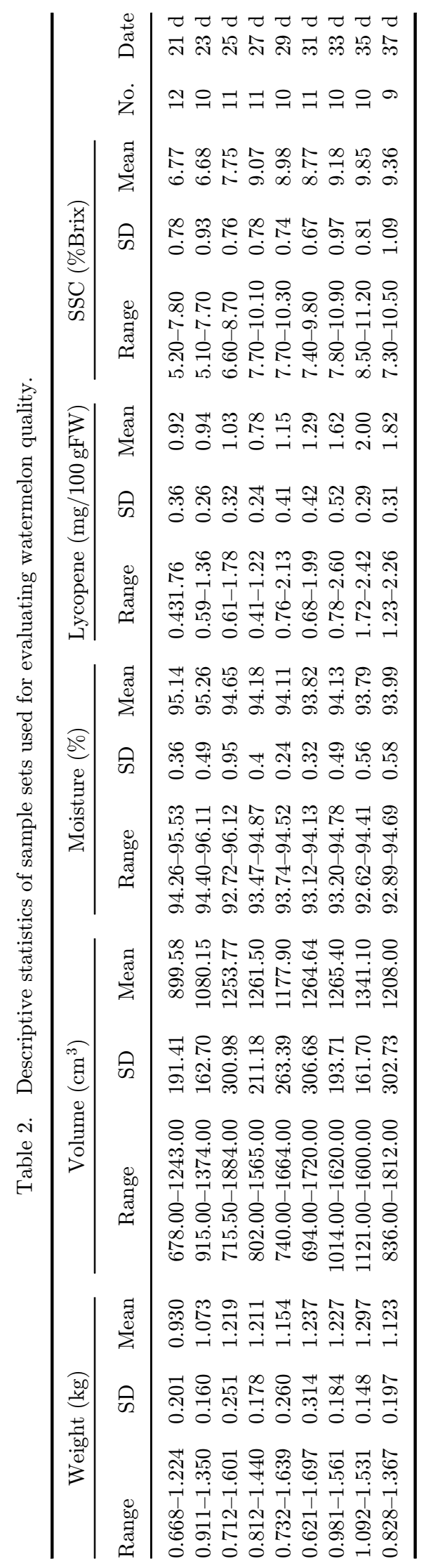




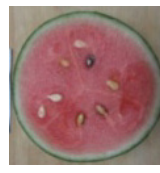

$21 \mathrm{~d}$

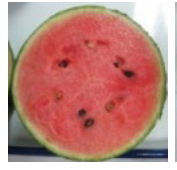

$31 \mathrm{~d}$

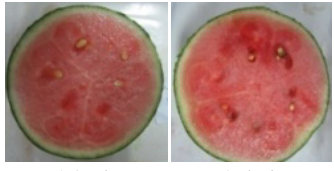

$23 \mathrm{~d}$

$25 \mathrm{~d}$

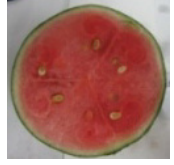

$27 \mathrm{~d}$

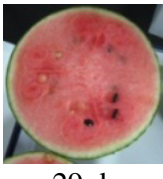

$29 \mathrm{~d}$
Fig. 2. Texture and color development of the watermelon varieties during the ripening process from $21 \mathrm{~d}$ to $37 \mathrm{~d}$ after pollination.

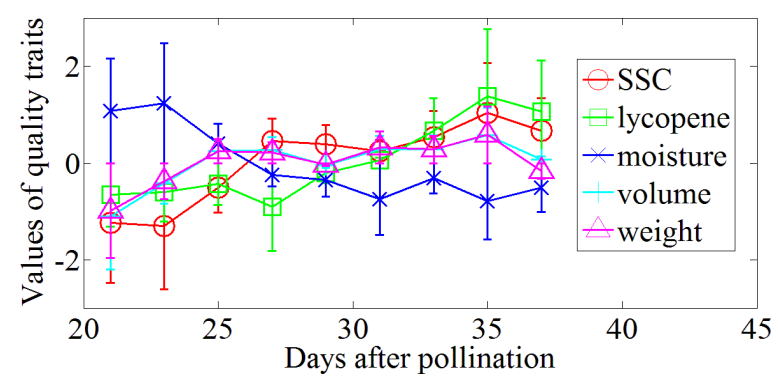

Fig. 3. Change of the average quality traits after standardized with error bars of $95 \%$ confidence interval during ripening period of watermelon.

spectroscopy was more convenient and lower cost than those independent element models.

As we have seen, $35 \mathrm{~d}$ after pollination had the best taste quality because of the biggest concentration of SSC and lycopene with lower moisture. It also can be found that the photo of $35 \mathrm{~d}$ was proper red pulp and black seed in Fig. 2. If we can predict the fully mature date by quality traits accurately, the picking date just moves up about three days.

\subsection{Prediction of chemical parameters by VIS-NIR spectroscopy}

The average absorption spectra for watermelon at different ripening stages were very similar to each other shown in Fig. 4(a), except that the spectrum of samples in $31 \mathrm{~d}$ shifted sharply according to the heavy rains.

Figure 4(b) showed that the spectra were in fact dominated by absorption peaks at 565 and $700 \mathrm{~nm}$ for prediction of lycopene, and the wavelength range of 525-625 nm were important in lycopene sensing. ${ }^{18}$ The peak value of $565 \mathrm{~nm}$ was very small

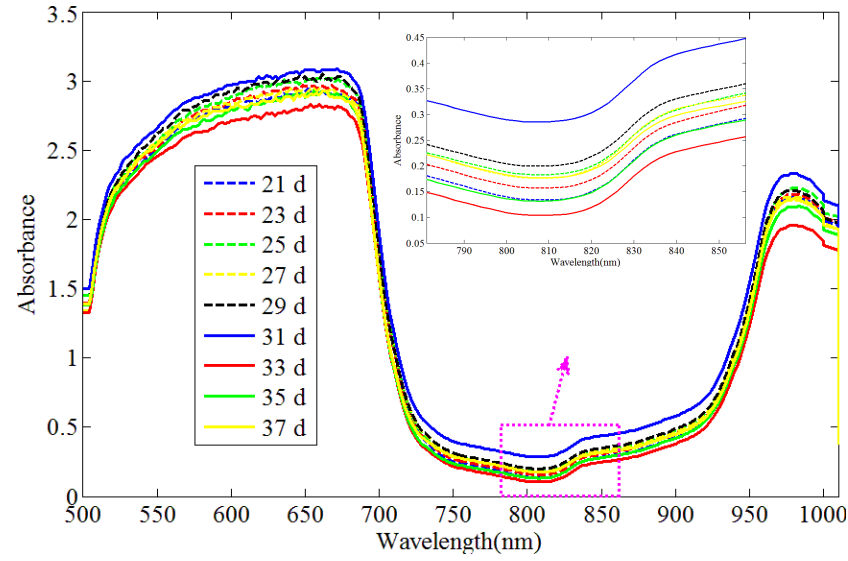

(a)

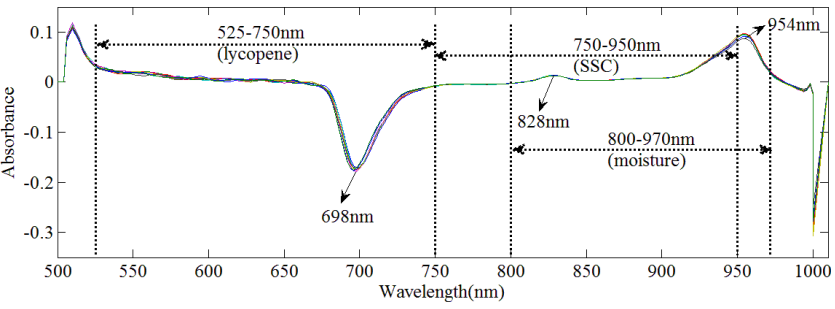

(b)

Fig. 4. The VIS-NIR diffuse transmittance spectra of watermelon (each spectrum was the average of all the samples at every picking date). (a) Typical original spectra for watermelon; (b) Watermelon spectra with first derivative.

and almost flat. Absorbance values at $698 \mathrm{~nm}$ were thus significant in lycopene index calculation. There was an absorption peak with second overtone bands of the NH-bonds and CH-bonds at $832 \mathrm{~nm}$, and it has been used to determine the firmness of fruits. ${ }^{19}$ The peak at $828 \mathrm{~nm}$ near $832 \mathrm{~nm}$ showed characteristic stretching vibration to develop models for detecting chemical concentration. One typical water absorption band with overtone band of the $\mathrm{OH}$-bonds was at $970 \mathrm{~nm} .{ }^{20}$ Due to the interaction between water bands and absorption bands of other constituents, shifts in absorption wavelengths should be taken in account. ${ }^{21}$ In part, $954 \mathrm{~nm}$ was sensitive to the change of moisture content.

According to the already known variability on watermelon constituents during the maturation period, the data matrix became representative of the expected variation on the related spectra. Since PLS regression model included all wavelengths including these bands, the PLS model provided acceptable precision of SSC, lycopene and moisture prediction (summarized in Tables 3 and 4). The 
Table 3. Statistics of chemical parameters of watermelon samples in calibration and prediction sets, respectively.

\begin{tabular}{lcccccccc}
\hline & \multicolumn{3}{c}{ Calibration $(n=70)$} & & \multicolumn{3}{c}{ Validation $(n=24)$} \\
\cline { 2 - 4 } \multicolumn{1}{c}{ Index } & Mean & SD & Range & & Mean & SD & Range \\
\hline SSC (\%Brix) & 8.6 & 1.303 & $5.5-11.2$ & & 7.9 & 1.476 & $5.1-10.3$ \\
Lycopene (mg/100 gFW) & 1.22 & 0.526 & $0.43-2.60$ & & 1.38 & 0.528 & $0.41-2.36$ \\
Moisture (\%) & 94.36 & 0.728 & $92.62-96.12$ & & 94.33 & 0.733 & $93.43-95.61$ \\
\hline
\end{tabular}

Table 4. Results of NIR calibration and validation performance for nondestructive quality assessment of watermelon.

\begin{tabular}{|c|c|c|c|c|c|c|c|c|}
\hline Index & Pretreatments & $\begin{array}{l}\text { Wavelength } \\
\text { range }(\mathrm{nm})\end{array}$ & Factors (LV) & $R c$ & RMSEC & $R p$ & RMSEP & $\mathrm{RPD}$ \\
\hline $\mathrm{SSC}(\%$ Brix $)$ & SG filt $(7,3)+1 \mathrm{D}$ & $750-950$ & 8 & 0.903 & 0.572 & 0.862 & 0.717 & 1.83 \\
\hline Lycopene (mg/100 gFW) & $\mathrm{SG}$ filt $(23,3)+2 \mathrm{D}$ & $525-750$ & 8 & 0.845 & 0.266 & 0.751 & 0.439 & 1.13 \\
\hline Moisture (\%) & SG filt $(7,3)+2 \mathrm{D}$ & $800-970$ & 10 & 0.917 & 0.280 & 0.939 & 0.276 & 2.79 \\
\hline
\end{tabular}

SG: Savitzky-Golay; 1D: first derivative; 2D: second derivative.

calibration models for all watermelon samples indicated a high correlation between the VIS-NIR spectra and the measured values including SSC, lycopene and moisture. When compared to the results of lycopenen model reported in Table 4, the higher prediction capability of moisture and SSC models were seen owing to its NIR sensitive characteristic and spectral responsibility, especially water to near-infrared spectroscopy. And yet the absorption of lycopene responded mostly in the range of visible light with lower signal-to-noise ratio. Compared to results in other papers, these models performed with higher precision but worse robustness. It is mainly because of insufficient number of samples. And this will be the considered issue to solve in the future.

\subsection{Prediction of physical parameters by $X$-ray image}

Baseline correction combined with parameter setting was needed to acquire X-ray image. Each image (0-255 grayscale) was also automatically saved as a gray value document to $2^{14}$ grayscale, an order of magnitude better than the image could achieve. Thus, the gray values in documents were applied to calculate the regression equation.

To fix watermelons on the conveyer belt when moving, an adjusted base was installed below samples. Interception vertical gray values across the centroid of watermelon X-ray image (Fig. 5) was useful to determine threshold value 1600 by the inflection point and eliminate interference of the base as well.

All the pixels below gray value threshold 1600 were extracted to sum. The range of the pixel sum was 74198-207392, and standard deviation was 30312. Linear fitting was built between sum of pixels and volume or weight respectively. The models of volume and weight were established shown in Figs. 6 and 7.

X-ray image cannot determine fruit volume directly, but it can give a measurement of the X-ray penetration capability which was influenced by density of the material. Associated with the relatively stable density of watermelon during the maturity stage, the sum of extracted pixels below threshold 1600 gray value was performed well to predict volume by linear regression $\left(R^{2}=9858\right)$. The average relative deviation was $2.14 \%$ between real values and predicted values of volume.

Weight is another physical parameter that cannot be measured directly by X-ray image, but can be quantified indirectly by measuring water content and dry matter which influenced the absorbility of X-ray. This decrease in water content and increase in dry matter as the fruit ripening contributed most significantly to the weight of the fruit. A calibration model for weight was developed with excellent predictability $\left(R^{2}=0.9927\right.$; average relative deviation $\left.=3.80 \%\right)$. 
S. Qi et al.

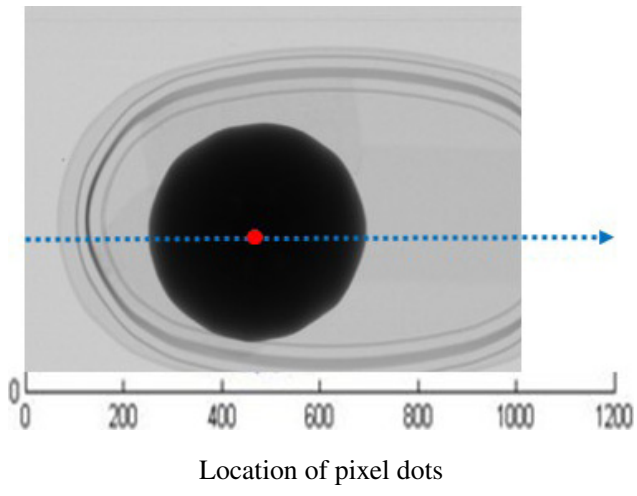

(a)

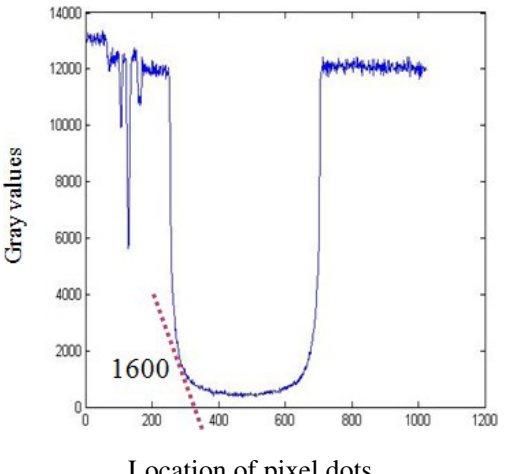

(b)

Fig. 5. Original X-ray image of one sample. (a) X-ray image of watermelon and its base; (b) The plot of gray values by intercepting vertical image $\mathrm{A}$ across the centroid of watermelon.

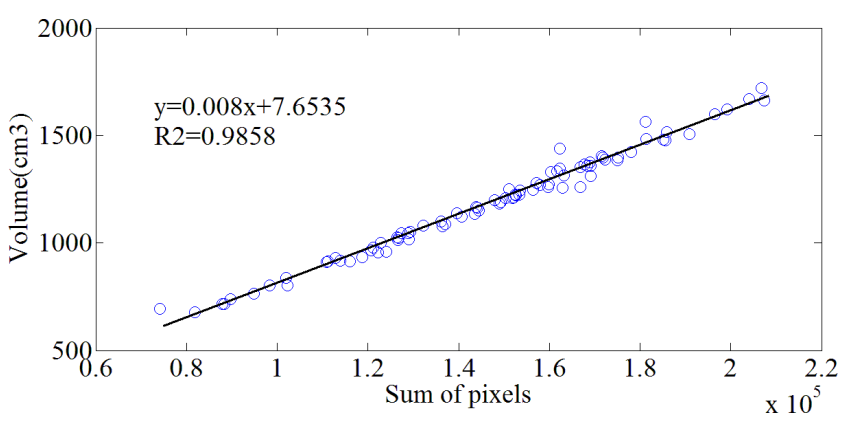

Fig. 6. The result of calibration model on volume.

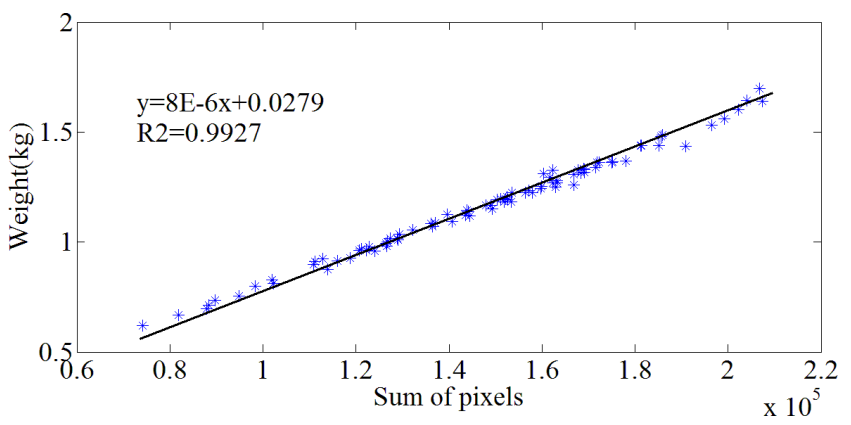

Fig. 7. The result of calibration model on weight.

\subsection{Factor analysis for the comprehensive indicator}

Figure 3 showed that all the traits changed in their own ways during ripening process of watermelon, and thus the evaluation model with single indicator was not convincible enough to distinguish quality of watermelons. In addition, significant interactive effects of these indexes were obtained according to Pearson correlation analysis (Table 5). It was necessary to do factor analysis to build the
Table 5. Pearson correlation coefficient of each two traits.

\begin{tabular}{lrcccc}
\hline & \multicolumn{5}{c}{ Index } \\
\cline { 2 - 6 } Index & SSC & Lycopene & Moisture & Volume & Weight \\
\hline SSC & 1 & - & - & - & - \\
Lycopene & $0.521^{\mathrm{a}}$ & 1 & - & - & - \\
Moisture & $-0.775^{\mathrm{a}}$ & $-0.496^{\mathrm{a}}$ & 1 & - & - \\
Volume & $0.382^{\mathrm{a}}$ & $0.281^{\mathrm{b}}$ & $-0.393^{\mathrm{a}}$ & 1 & - \\
Weight & $0.327^{\mathrm{a}}$ & 0.163 & $-0.339^{\mathrm{a}}$ & $0.950^{\mathrm{a}}$ & 1 \\
\hline
\end{tabular}

${ }^{\mathrm{a}}$ significant correlation at the level of 0.01 ; ${ }^{\mathrm{b}}$ significant correlation at the level of 0.05 .

comprehensive indicator for better precision of prediction model.

Principal component analysis (PCA) was performed as the extraction method. The PCs 1 and 2 accounted for $83.515 \%$ of the variation. The PC1 explained $56.986 \%$ of the total variance between indicators, whereas the PC2 explained $26.530 \%$ of the variation. Figure 8 revealed that it was reasonable to choose $\mathrm{PC} 1$ and $\mathrm{PC} 2$ instead of the whole information.

In order to improve explanatory of selected factors, the data matrix was rotated by varimax with Kaiser normalization method converged in 25 iterations (Fig. 9). The rotated PC plot of PCA illustrated the large variability of watermelon in variety maturity periods to cover most of the possible range of quality traits. PC1 discriminated the fruits mainly according to chemical parameters for internal quality, and $\mathrm{PC} 2$ was representative for physical variations for the size of fruit. Then component score coefficient was calculated by regression method showed in Table 6. Due to the component 


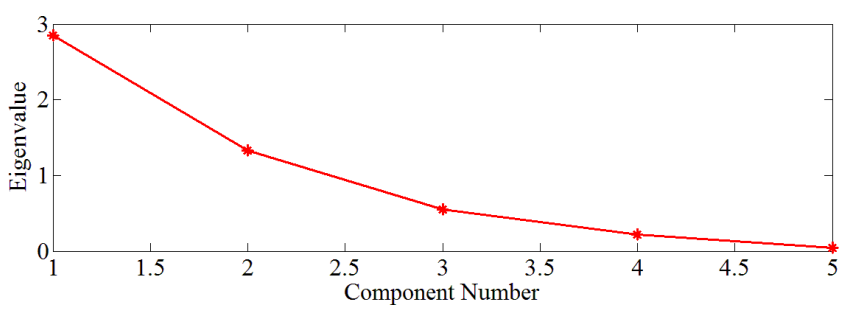

Fig. 8. Scree plot of total variance explained.

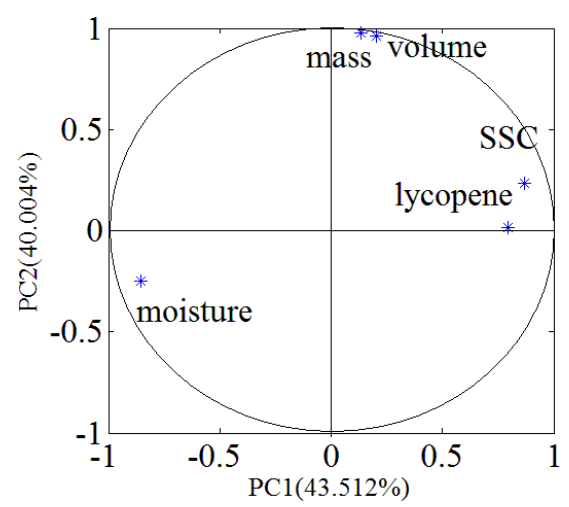

Fig. 9. The plot of rotated component matrix.

Table 6. Component score coefficient matrix.

\begin{tabular}{lrrr}
\hline & \multicolumn{3}{c}{ Component } \\
\cline { 2 - 4 } \multicolumn{1}{c}{ Index } & \multicolumn{1}{c}{$\mathrm{F} 1$} & \multicolumn{1}{c}{$\mathrm{F} 2$} & \multicolumn{1}{c}{$\mathrm{F}$} \\
\hline $\mathrm{SSC}(\%$ Brix $) \rightarrow \mathrm{X} 1$ & 0.414 & -0.040 & 0.197 \\
Lycopene $(\mathrm{mg} / 100 \mathrm{~g}) \rightarrow \mathrm{X} 2$ & 0.417 & -0.151 & 0.145 \\
Moisture $(\%) \rightarrow \mathrm{X} 3$ & -0.402 & 0.027 & -0.197 \\
Volume $\left(\mathrm{cm}^{3}\right) \rightarrow \mathrm{X} 4$ & -0.085 & 0.514 & 0.202 \\
Weight $(\mathrm{kg}) \rightarrow \mathrm{X} 5$ & -0.124 & 0.535 & 0.192 \\
\hline
\end{tabular}

Extraction method: Principal component analysis.

Rotation method: Varimax with Kaiser normalization

$$
\begin{aligned}
\mathrm{F} 1= & 0.414 \mathrm{X} 1+0.417 \mathrm{X} 2-0.402 \mathrm{X} 3-0.085 \mathrm{X} 4 \\
& -0.124 \mathrm{X} 5=\sum_{i=1}^{5} a_{i} X_{i} \\
\mathrm{~F} 2= & -0.040 \mathrm{X} 1-0.151 \mathrm{X} 2+0.027 \mathrm{X} 3+0.514 \mathrm{X} 4 \\
+ & 0.535 \mathrm{X} 5=\sum_{i=1}^{5} b_{i} X_{i} \\
\mathrm{~F}= & \sum_{i=1}^{5} c_{i} X_{i}, \quad \text { and } \\
c_{i}= & \frac{2.176}{2.176+2.000} \times a_{i}+\frac{2.000}{2.176+2.000} \times b_{i}
\end{aligned}
$$

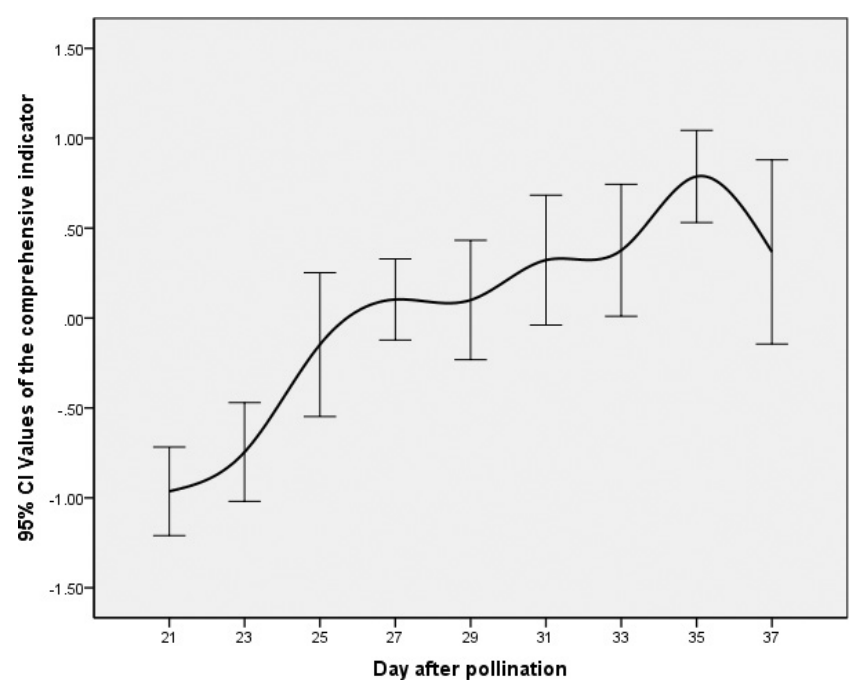

Fig. 10. Change of the comprehensive indicator with error bars of $95 \%$ confidence interval during ripening period of watermelon.

score covariance matrix, PC1 and PC2 had no relationship with each other. Thus, to establish the comprehensive indicator, the rotation eigenvalues 2.176 and 2.000 of $\mathrm{PC} 1$ and $\mathrm{PC} 2$ respectively were presented to obtain coefficients of the total factor. The formula was showed following the Table 6 .

The change of watermelon quality at different ripening stages was presented in Fig. 10 clearly and reasonably. It was easy to distinguish the maturity by the value of the comprehensive indicator directly. $35 \mathrm{~d}$ after pollination was the day that Jingxiu matured completely. In this study the fullspectrum PLS model using 10 factors was developed using raw spectra with first derivative processing in the range from 525 to $970 \mathrm{~nm}$.

From Fig. 11, it can be seen that this model have a better precision and robustness than the singletrait models, and the best result was obtained by

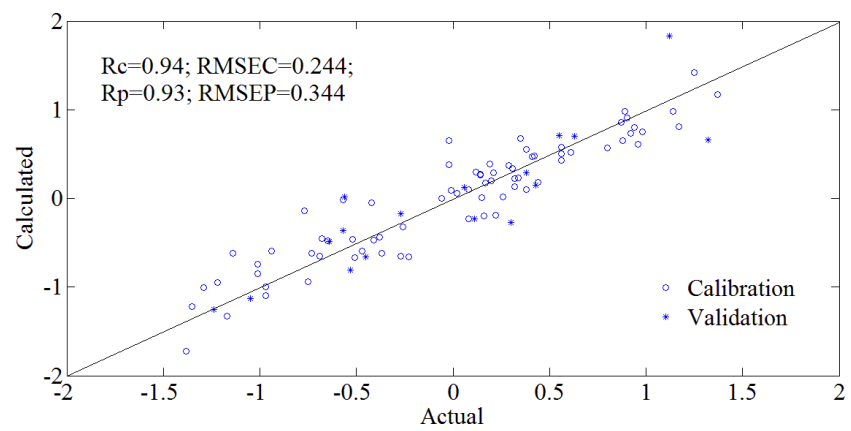

Fig. 11. The quantitative model of calibration and validation sets on the comprehensive indicator. 
selecting the comprehensive indicator with $R c$ of 0.94, RMSEC of $0.244, R p$ of 0.93 , RMSEP of 0.344 , RPD of 2.00. For this study, it was based on a small amount of samples and single cultivar within a 17-day period. It is assumed that a bigger sample set, a more sampling cultivar and a variable season, as used in our study, might result in better model performance.

\section{Conclusion}

On intact watermelon, VIS-NIR spectroscopy could be used to predict SSC, lycopene and moisture, and $\mathrm{X}$-ray image could be used to detect volume and weight nondestructively. But, the evaluation of single trait seemed not be sufficiently accurate and not convincible enough to assess a natural product. Given the interplay of different indexes and its complex performances during the maturity process, the comprehensive indicator was established by factor analysis to improve the prediction precision of rapid noninvasive technique. In the future we plan to check the robustness of this model on other watermelons, including different cultivars, harvested over different years and cultivated in different orchards. And it was to monitor at the calyx, whose rind was the thinnest, whose location was most stable to acquire spectra and who was most sensitive to growth process of watermelon. According to its specific characteristics, it looks possible to apply this method on large size watermelon. In addition, the comprehensive model using portable VIS-NIR spectrometer appeared already as a suitable method for appraising watermelon quality at different ripening stages on the plant. Then discriminating the picking date will be possible depending on theory other than experience.

\section{Acknowledgments}

This study was supported by the national natural science foundation (NSFC) (NO. 31071555) to provide financial support and the earmarked fund for Modern Agro-industry Technology Research System (NO. CARS-26-22) to measure reference values using traditional methods.

\section{References}

1. J. B. Li et al., "A comparative study for the quantitative determination of soluble solids content, $\mathrm{pH}$ and firmness of pears by Vis/NIR spectroscopy," $J$. Food Eng. 116(2), 324-332 (2013).

2. B. B. Wedding et al., "Effects of seasonal variability on FT-NIR prediction of dry matter content for whole Hass avocado fruit," Postharvest Biol. Technol. 75, 9-16 (2013).

3. A. Morales-Sillero et al., "Feasibility of NIR spectroscopy for non-destructive characterization of table olive traits," J. Food Eng. 107(1), 99-106 (2011).

4. Y. Palapol et al., "Colour development and quality of mangosteen (Garcinia mangostana L.) fruit during ripening and after harvest," Postharvest Biol. Technol. 51(3), 349-353 (2009).

5. T. Sun, K. Huang, H. R. Xu, Y. B. Ying, "Research advances in nondestructive determination of internal quality in watermelon/melon: A review," J. Food Eng. 100, 569-577 (2010).

6. D. F. Jie et al., "Variable selection for partial least squares analysis of soluble solids content in watermelon using near-infrared diffuse transmission technique," J. Food Eng. 118, 387-392 (2013).

7. K. S. Chia, H. A. Rahim, R. A. Rahim, "Evaluation of common pre-processing approaches for visible (VIS) and shortwave negar infrared (SWNIR) spectroscopy in soluble solids content (SSC) assessment," Biosyst. Eng. 115, 82-88 (2013).

8. S. Bureau et al., "Rapid and non-destructive analysis of apricot fruit quality using FT-near-infrared spectroscopy," Food Chem. 113, 1323-1328 (2009).

9. E. D. Louw, K. I. Theron, "Robust prediction models for quality parameters in Japanese plums (Prunus salicina L.) using NIR spectroscopy," Postharvest Biol. Technol. 58, 176-184 (2010).

10. D. Dimitrovski et al., "The concentration of translycopene in postharvest watermelon: An evaluation of analytical data obtained by direct methods," Postharvest Biol. Technol. 58, 21-28 (2010).

11. H. W. Zhao, D. H. Han, S. H. Song, D. Chang, "Screening of maturity characterization factors for mini watermelon fruit," Trans. CSAE 28(17), 281286 (2012).

12. Y. Y. Li, H. W. Zhao, D. Chang, D. H. Han, "Maturity qualitative discrimination of small watermelon fruit," Spectrosc. Spect. Anal. 32(6), 1526-1530 (2012).

13. G. P. Moreda et al., "Non-destructive technologies for fruit and vegetable size determination - a review," J. Food Eng. 92, 119-136 (2009).

14. X. D. Sun et al., "A fast online method to measure and calculate the apple's cubage based on X-ray image," Acta Optica Sinica 27(11), 2096-2100 (2007).

15. D. H. Han et al., "Information collection of mini watermelon quality using near-infrared non-destructive detection," Trans. CSAE 44(7), 174-1788 (2013). 
16. T. Ignat et al., "Nonlinear methods for estimation of maturity stage, total chlorophyll, and carotenoid content in intact bell peppers," Biosyst. Eng. 114, 414-425 (2013).

17. L. Jarquin-Enriquez et al., "Lycopene content and color index of tomatoes are affected by the greenhouse cover," Sci. Hortic. 155, 43-48 (2013).

18. R. Choudhary et al., "Rapid estimation of lycopene concentration in watermelon and tomato puree by fiber optic visible reflectance spectroscopy," Postharvest Biol. Technol. 52, 103-109 (2009).
19. Y. K. Peng, R. F. Lu, "Analysis of spatially resolved hyperspectral scattering images for assessing apple fruit firmness and soluble solids content," Postharvest Biol. Technol. 48, 52-62 (2008).

20. B. M. Nicolai et al., "Nondestructive measurement of fruit and vegetable quality by means of NIR spectroscopy: A review," Postharvest Biol. Technol. 46(2), 99-118 (2007).

21. R. Giangiacomo, "Study of water-sugar interactions at increasing sugar concentration by NIR spectroscopy," Food Chem. 96, 371-379 (2006). 